\title{
Observed Periodic Phenomena
}

\author{
D. Baade \\ European Southern Observatory, Karl-Schwarzschild-Str. 2, \\ D-85748 Garching b. München, Germany
}

\begin{abstract}
The wide range of periodic and cyclic variabilities commonly observed in Be stars and their circumstellar disks is reviewed. Many of them are related to the basic nonradial pulsation, the effects of which may range from the photosphere to the disk. Through pulsation-triggered outbursts, some stars may owe their Be characteristics largely to their intrinsic variability. However, because late-type Be stars do not normally show detectable short-periodic variability, this is probably not true of all Be stars. Comparisons are made with other variable early-type stars.
\end{abstract}

\section{Introduction}

In astronomy, the only substitute for laboratory experiments are observations of variability and different evolutionary phases. Especially periodic variations often offer a considerable diagnostic potential. This paper will, as its first purpose, take this fact to its limits and not only review the rather numerous variabilities of Be stars but try to argue that Be stars are Be stars, because they are (periodically) variable. Since the Be phenomenon may in more subtle forms occur also in other hot stars (cf. Sect. 5.) and Be stars are the most complex variables among them, Be stars might serve as optimal hot-star laboratories.

Although it may be tempting to perceive $\mathrm{Be}$ stars as objects of unique peculiarity, Occam's Razor suggests that rather the applicability of the following working hypotheses should be exhausted: (1) the physics of Be stars is not any different from the one of early-type stars in general, (2) other early-type stars contain ready-to-use building blocks for the basic model of the Be phenomenon. Accordingly, one would try to build a model of the Be phenomenon from largely unmodified template components established for other early-type stars and only combine them differently. The second purpose of this paper is, therefore, to show that in this way interesting and, so far, self-consistent progress can be made.

Nevertheless, for both purposes the final observation is still to be obtained.

\section{Clocks and Periods}

There are numerous potential clocks in Be (and other) stars: stellar pulsation and rotation, orbital motion, disk precession, disk oscillations, magnetic activity cycles, outbursts, and orbiting clouds, comets, and planets. They have different degrees of stability. More importantly, their readings can be seriously affected by the ticking of the respective other clocks. Most worrisome are the various 
aperiodic phenomena (cf. Smith, these proc.). Therefore, utmost care has to be applied when searching for periods and assessing the results. In addition, observations have to be planned keeping the requirements for the subsequent time series analysis in mind. That is, not only have the data to be analysed in Fourier space, but observing projects have to be layed out in Fourier space: It is well possible that the Be phenomenon can be decoded in Fourier space only.

This raises the question of what can eventually be called a period. Mathematically, the answer is easy: a process is periodic, if and only if it repeats forever without changes. For observers with finite life expectancy and evolving stars, this is not a realistic requirement. The proper balance between uncritical period hunting and hair splitting can, therefore, be a delicate one. However, the accuracy of a period is always less decisive than its attribution to the correct physical process. For instance, Kepler's laws initially led to much inferior ephemerides for the planets than did the previous epicycle model. And yet, it was Kepler's laws that provided the proper explanation of the orbital motions.

To this end, it is essential that the data to be collected permit physical diagnostics to be extracted. For instance, single-channel photometry and plain radial velocity measurements are mostly insufficient for this purpose, except that reliable multiperiodicities may be important to exclude particular models.

\section{Periodic Variabilities of Be Stars}

Proceeding from the circumstellar envelope and longer timescales towards the central star and shorter timescales, this section examines the clocks so far known of Be stars. Although there are Be stars, which owe their disk to mass transfer in a binary, orbital effects (e.g., Rivinius \& Štefl, these proc.) are excluded, because binarity is not a core property of the Be phenomenon (Baade 1992).

\subsection{Slow $\mathbf{V} / \mathrm{R}$ variations}

Variations of the intensity ratios $(\mathrm{V} / \mathrm{R})$ of the violet and red components of double-peaked emission lines on a timescale of years are among the longestknown variabilities of Be stars. They arise from velocity fields and non-axisymmetric density distributions in the circumstellar disk. Lines from transitions favoured by different physical conditions may differ in their $V / R$ ratios and even in the sign of $\log V / R$. The periodic component of this slow $V / R$ variability appears to be well explained by global one-armed oscillations (cf. Stee, Okazaki, these proc.). The vulnerability of the disk structure by aperiodic and periodic activities of the central star makes these long-term $V / R$ variations very poor clocks. They are probably only a secondary symptom of the Be phenomenon.

A circumstellar disk is a key characteristic of Be stars. In other single $O B$ stars, disks have only been inferred for Herbig Ae/Be stars (Böhm, these proc.), where, however, they are remnants of the star formation process, and $B[e]$ stars (Zickgraf, these proc.), which on average are much more luminous than Be stars.

\subsection{Discrete absorption components of UV resonance lines}

Similar to more luminous OB stars (e.g., Prinja 1998), discrete absorption components ("DACs") of UV resonance lines are common in Be stars (but not in 
B stars; Underhill \& Doazan 1982). However, the contribution of DACs to the total wind absorption is usually larger in Be stars. It is conceivable in extreme cases that they may account for the entire wind absorption. DACs probably reflect density variations, which may be due to intrinsic wind stabilities or triggered by the variability of the underlying star or both (Owocki 1999). Since non-Be dwarfs and giants generally do not display DACs, DACs seem to be integral part of the Be phenomenon. It is, therefore, regrettable that the repetitiveness of DACs in Be stars is much less well studied than in OB supergiants (cf. Kaufer, these proc.). The observed timescales range from days to more than a year (Peters 1999).

\subsection{Outbursts}

Contrary to OB supergiants (e.g., Prinja 1998), the mass loss rates of Be stars are highly variable as deduced from UV resonance lines, which probably preferentially sample the polar directions (Grady et al. 1987), as well as from disk lines, which reflect more equatorial directions. Only over the past 10-15 years has this fact led to the widespread notion that much of this activity is due to discrete events or outbursts. Since similar activities have not been observed in less evolved, non-emission line B stars, this is most likely another characteristic of the Be phenomenon. Although in $\mathrm{OB}$ supergiants the relative variability of the mass loss is much smaller, the absolute variation is easily of a similar order of magnitude. Therefore, this property, too, may be shared by other OB stars.

There are numerous photometric, spectroscopic, and polarimetric examples of single outbursts in the literature. However, two major databases are probably much more effective in demonstrating the statistical relevance of this behaviour: (i) Hipparcos detected sudden brightenings in a fair fraction of the Be stars observed (Hubert \& Floquet 1998). At times, these outbursts seem to repeat, possibly cyclically, after typically $100-300 \mathrm{~d}$. (ii) The MACHO project discovered many so-called "bumpers" in the Large Magellanic Cloud (Cook et al. 1995). They fall into two different categories distinguished by hump-like and rectangular light curves, respectively. When an identification was attempted, many of the stars concerned turned out to be Be stars. Given the good sampling, the long time baseline, and the low metallicity of the LMC, it would be very desirable to analyse this database for any repetitive outbursts.

With only scattered observations, the implicit assumption has often been that outbursts of $\mathrm{Be}$ stars are stochastic events. However, in $\mu$ Cen, intensive spectroscopic long-term monitoring revealed (Rivinius et al. 1998a; Stefl \& Rivinius, these proc.) that outbursts repeat every $29 \mathrm{~d}$ and $54 \mathrm{~d}$. Hubert \& Floquet (1998) derived from Hipparcos data a repetition timescale of $20 \mathrm{~d}$. Since the double periodicity went unnoticed in this dataset, this shorter timescale is perfectly consistent with the two other ones. More recent spectroscopy by Tubbesing et al. (these proc.) identified 28 Cygni as a promising second candidate for periodic outbursting with a period of $18 \mathrm{~d}$.

Rivinius et al. (1998a) used their spectra of $\mu$ Cen to establish also a detailed temporal outburst profile, which comprises four main phases (see also Sttefl \& Rivinius, these proc.). Unpublished observations by the same authors of a few other Be stars suggest that the same outburst pattern may be relatively com- 
mon. With its sudden onset and slower decay, it corresponds more nearly to the triangular MACHO light curves and closely resembles those from HIPPARCOS.

\subsection{Transient periods}

In a couple of stars with long series of spectroscopic observations, so-called transient periods have been found (cf. Štefl et al., these proc.). Their values are typically within $10 \%$ of the primary photospheric period. The variations are often rather of a more cyclic nature lasting for a couple of weeks to months, probably reside mainly in the upper photosphere, and are linked to outbursts. In different outburst cycles, transient periods are only reproduced to within $\sim 10 \%$. So far, only one transient period has been found at any one time. This phenomenon does not seem to have known counterparts in other types of stars.

\subsection{Rapid $\mathbf{V} / \mathbf{R}$ variations}

In many $\mathrm{Be}$ stars, the $\mathrm{V} / \mathrm{R}$ ratio of Balmer and $\mathrm{HeI}$ emission lines varies at times also on a timescale of order one day. Although this symptom seems to be rather uniform (but with line-to-line differences), it is probably associated with different physical processes because:

1. in its simplest form, the $V / R$ ratio has the period of the low-order line-profile variability of the central star,

2. the $V / R$ ratio is one of the line profile characteristics, which are particularly affected by transient periods,

3. rapid V/R variations also are an integral characteristic of many outbursts (Rivinius et al. 1998a; Štefl \& Rivinius, these proc.).

Because transient periods seem to occur preferentially close to outbursts, the last two items may not really be different (cf. Sect. 4.2.). For the first one, Penrod (1986) suggested a "lighthouse effect" of a photospheric temperature modulation on the disk. With current codes for the disk ionisation structure (e.g., Millar, these proc.), it should be relatively straightforward to verify or reject this idea. However, this variability may also only be apparent and actually due to the variability of the underlying photospheric profile (Maintz et al., these proc.).

\subsection{Circumstellar Balmer discontinuity variations}

A possibly related effect is the variability of the circumstellar Balmer discontinuity. Although it has so far been studied only in $\eta$ Cen (Štefl et al. 1995 and in preparation), the possible detection of the stellar period $(0.64 \mathrm{~d})$ in the circumstellar Balmer disconuity would be the strongest hint to date of a short-periodic interaction between photosphere and near-circumstellar environment.

Such a variability (and, perhaps, the rapid $V / R$ variations) may offer a possibility to probe the disk structure radiatively. Conversely, they could serve as a "thermometer" for the calibration of stellar temperature modulations.

\subsection{Nonradial pulsation or (magnetically controlled) rotational mod- ulation?}

At the previous major Be-star conference, this debate about the explanation of the short-periodic photometric and line profile variability $(l p v)$, which, except for the later spectral sub-classes, is found in virtually all Be stars, was still 
one of the most controversial points (cf. Baade \& Balona 1994). Eleven poster papers presented at the present conference and reporting multiply periodic $l p v$ leave no doubt that many Be stars do pulsate nonradially. Moreover, state-ofthe-art modeling now permits observed line profiles to be fitted with physical models as opposed to the previously often used qualitative comparison between observations and purely parametric computations.

The diversity of details of the $l p v$, that can be reproduced with pulsational eigenfunctions and physical model atmospheres appropriate for rapidly rotating stars, is remarkable (Maintz et al., these proc.; Rivinius, these proc.), even though such fits are not unique at currently affordable signal-to-noise ratios and spectral resolution. The line profile modulation is generally the most pronounced in Be stars with intermediate to low $v \sin i$, and the nonradial pulsation (NRP) model passed the various associated, very stringent tests with considerable success:

- red-to-blue moving features: These are due to the latitudinal velocity component $\left(v_{\theta}\right)$ of $g$-modes, which in pole-on stars is still visible in the quadrant "behind" the rotation axis and above the stellar equator.

- narrow absorption spikes, which occur periodically at $\sim \pm 0.7 v \sin i$ : In poleon stars, they are due to the temporary coincidence of the line-of-sight component of $v_{\theta}$ of low-order $g$-mode velocity fields with the rotational radial velocity of a major portion of the stellar surface.

- nearly straight ramps accompanying spikes in the respective opposite line wing: They, too, are an effect of $v_{\theta}$ in $g$-mode velocity fields, which add to the high end of the rotational velocity field.

- combinations of very low photometric amplitudes and large-amplitude lpv: Even for low-order modes, there are major cancellation effects between areas of low and high temperature, while net geometric distortions are still low (Townsend 1997).

By contrast, all rotational models proposed to date (cf. Balona, these proc.) expose significant weaknesses when they are forced to deliver a unified explanation of Be stars seen at any inclination angle. Since this is true even for single-periodic stars, the multiperiodicity, which is now being found in more and more Be stars, makes it safe to conclude that the contribution of co-rotating structures to the explanation of the short-periodic variability of Be stars is at most minor.

This does not rule out the main ingredient to most rotational models, namely magnetic fields. Even the complete lack of direct evidence for magnetic fields in conventional Be stars (Mathys \& Smith, Smith, these proc.) does not eliminate the possibility of magnetic effects in addition to those of NRP. The correct explanation of the widespread short-periodic variability does not in any way imply that NRP is sufficient to explain also the Be phenomenon as a whole. However, the intriguing link between the beating of NRP modes and outbursts (Sect. 4.1.) in some Be stars may mean that for their being Be stars NRP is necessary. Therefore, the real question is not: NRP or magnetically controlled rotational modulation? but: What is the relative importance of these two kinds of processes in making a $B$ star a Be star?

It is not contradictory with this conclusion, nevertheless, to explore the limits of the explanatory capabilities of pure NRP models, so long as one is 
aware that these limits must exist but have yet to be identified: This approach simply requires the lowest degree of extrapolation from the current observations.

\section{Effects of Nonradial Pulsations}

Several conjectures about NRP-driven mass loss mechanisms have been proposed (e.g., Ando 1986, Osaki 1986, Penrod 1986) in an attempt to explain also the Be phenomenon at large. But none of them have found an observational confirmation. Very interestingly, recent observations suggest strongly that the to be explored limit to the explanatory power of the NRP model is indeed not somewhere in the photosphere but well above it.

\subsection{Outbursts}

\section{$\mu$ Centauri [and 28 Cygni]}

The 'Rosetta star' in such a circumstellar extension to the NRP model would be $\mu$ Cen (Rivinius et al. 1998b, 1998c), with 28 Cyg (Tubbesing et al., these proc.) apparently sharing many of its key properties:

- there are several closely spaced (by no more than a few percent) periods near $0.5 \mathrm{~d}$ [28 Cyg: $0.64 \mathrm{~d}$ ],

- the corresponding NRP modes have the same $\ell$ and $m$ [28 Cyg: ditto],

- the azimuthal mode order is low: $|m| \leq 2$ [28 Cyg: ditto],

- outbursts repeat every 29 and 54 days [ $28 \mathrm{Cyg}: 18$ days, to be confirmed], when two of the 0.5-d [28 Cyg: 0.64-d] modes interfere constructively,

- the superposition of modes with a combined amplitude lower than the beat amplitude of combinations, that do lead to outbursts, has no effect ( $\mu \mathrm{Cen}$ ),

- the beating of modes with different $\ell$ and/or $m$ is not accompanied by outbursts, even if the combined amplitude is large ( $\mu$ Cen).

It is quite plausible to infer from this list that an outburst requires a threshold in combined pulsational velocity to be exceeded and the geometry $(\ell$ and $m)$ of the co-added velocity fields to be identical. If NRP plays a role in triggering outbursts, both circumstances would probably ensure maximum effectiveness.

One may speculate that above this implied threshold the combined NRP waves become non-linear, at which point matter may be transported by them with their propagation velocity, which in the linear regime only is a phase velocity, i.e. no matter moves with this velocity. This is crudely comparable with the breaking of waves on the shore, which far away from it only undulate the surface of the water but on land may develop devastating power.

Such a simple model was recently proposed by Osaki (1999). He concludes that necessary conditions for the occurrence of outbursts are:

(a) a velocity amplitude above the limit of linearity,

(b) prograde modes $(m<0)$, because only then would any matter transported with the phase velocity of a wave move in the direction of the rotation,

(c) a small $|m|$, because the phase velocity of NRP waves is inversely proportional to $m$ so that for such modes the velocity kick would be largest,

(d) rapid rotation and a short period so that the sum of rotation and phase velocity exceeds the critical velocity, thereby enabling the ejection of matter. 
The proof that this mechanism is energetically effective enough to account for the magnitude of outbursts is still pending. The essence of the solution to the companion problem of the angular momentum transfer so that (part of) the ejecta can reach the disk may have been given by Kroll \& Hanuschik (1997).

The still unknown physical mechanism, which is triggered by the beating of NRP modes, may well include a magnetic component. The lacking detection of ordered magnetic fields (Mathys \& Smith, these proc.) and the apparent high degree of similarity both between various outbursts of $\mu$ Cen and between those of $\mu$ Cen and a few other Be stars (Sect. 3.3.) might suggest that any such fields would be diffuse and cover a large proportion of the stellar surface.

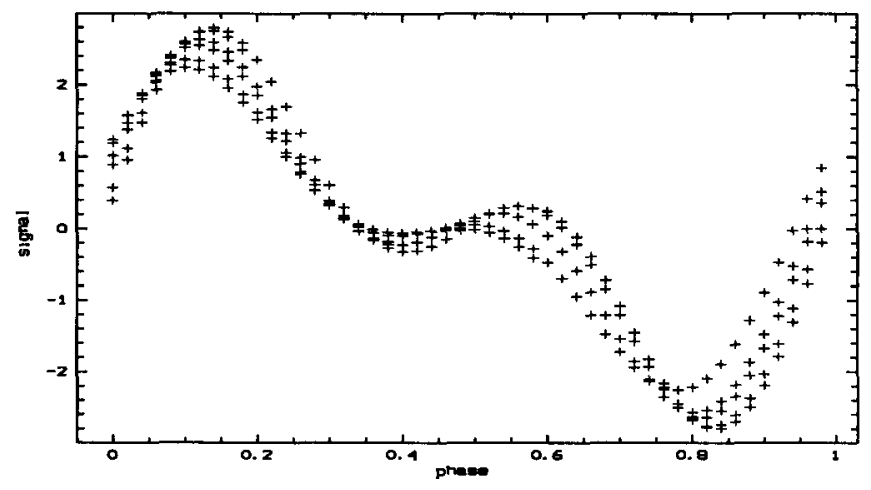

Figure 1. Schematic light curve constructed from four sinusoidal components with $P_{2}=0.5 P_{1}, P_{3}=0.985 P_{1}, P_{4}=0.5 P_{3}, A_{2}=A_{3}=$ $0.8 A 1, A_{4}=0.8 A_{3}$. Observations are assumed to be quasi-continuous over $31 \%$ of $P_{1}$, followed by a break of equal length. The signal is plotted versus phase of $P_{1}$. Note the basic similarity with the light curve of o And (Sareyan et al. 1998)

\section{Other stars?}

The slow, low- $\ell$ pulsations of Be stars are probably some $g$-type modes of high radial order. In theoretical models, classical $g$-modes have closely spaced periods and several such neighbouring modes would be expected to be excited simultaneously. It has, therefore, been puzzling that only one mode of a kind seemed to be excited in Be stars. Possibly the four 0.5-d periods in $\mu$ Cen, and also the regularity in their spacing, might offer a solution to this long-standing riddle. (Note that the multiperiodicity found so far in Be stars other than $\mu$ Cen [and $28 \mathrm{Cyg}$ ] is probably different as those variabilities belong to modes with different $\ell$ and/or $m$, which is also the case for the 0.28 -d and 0.5 -d periods in $\mu$ Cen.)

$\mu$ Cen was selected for intensive monitoring, because it was already known to undergo relatively frequent outbursts. If the outbursts in the HIPPARCos database (Hubert \& Floquet 1998) correspond to the same phenomenon, this choice was fortunate, since, among the stars with multiple outbursts, $\mu$ Cen is the one with the highest outburst frequency. Accordingly, if the more typical recurrence times of outbursts of 200-300 d correspond to beat periods of NRP modes, observations suitable for the separation of so closely spaced periods will 


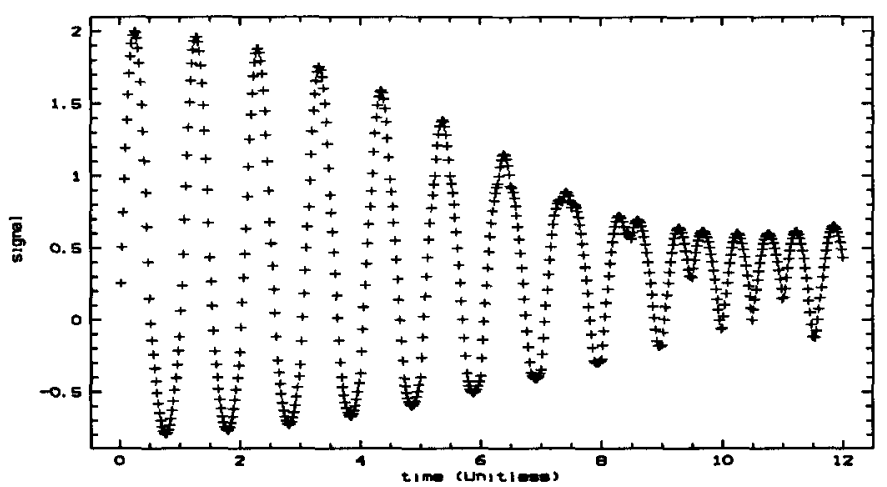

Figure 2. The beating of two light curves, which differ in their periods by $5 \%$, but otherwise are identical. For both the positive and the negative halfwave the curves are sinusoidal. However, the lower (negative) halfwave have been scaled by a factor of 0.4 . Note the progression (from left to right) from a single to a double waves at constant period with concommittant reduction of the amplitude

require significant efforts. Nevertheless, it would be very worthwhile to carry out such a test for at least one or two of these stars.

It could also be useful to re-analyze existing data and check whether by the admission of multiple, closely spaced periods some previously unsolved problems take on a different appearance. But it is mandatory to remember that various mathematical circumstances usually conspire to support spurious multiperiodic solutions so that critical physical, observational, and numerical judgement has to be applied before accepting them. Two specific examples of such a potential transformation of a problem are suggested in the following:

o And: This very intensively studied classical Be star features a double-wave light curve with strong season-to-season variations in amplitude and considerable cycle-to-cycle phase migration (Sareyan et al. 1998; Guerrero et al., these proc.). Formally, it is relatively straightforward to simulate the latter effect with two double-wave light cures with closely spaced periods (Fig. 1), which could also account for the long-term amplitude variation.

Double-wave light curves, although often less pronounced than in o And, are also seen in many other Be stars. In two cases, season-to-season changes between double- and single-wave light curve at constant period have been reported (Balona et al. 1991), which are difficult to understand in the framework of a single-mode pulsation. Both stars have in common that their amplitude is smaller when the light curve is of the double-wave type. These two phenomena can also be emulated under the assumption of two closely spaced periods (Fig. 2 ), if the light curves are, in addition, also non-sinusoidal. With only two known examples, the significance of such a comparison is, of course, extremely limited.

\subsection{Stellar-circumstellar synopsis, or putting it all together}

Having seen the bewildering manifold of periodic variabilities one by one, one is curious what they would look like if put all together. This is probably not 
any different from the wish to know how Be stars are distinguished from other B stars, i.e. the answer is still unknown. The picture, which is nevertheless sketched below, is therefore necessarily strongly simplified and in some parts a strong extrapolation of the established knowledge. Even if it is basically correct, it can probably account for Be stars of early spectral types only. Beyond $\sim \mathrm{B} 7$ the amplitude and/or incidence of periodic short-term variability drop below the current level of spectroscopic (Baade 1989) and photometric (Balona et al. 1992) detectability. Although observations have not (yet) provided compelling reasons to speak of more than one Be phenomenon, there is very probably more than one physical process - presumably even among the hotter Be stars - which may let a B star display what is currently called the Be phenomenon. The picture would presently look as follows:

Be stars are relatively hot and little-evolved, rapidly rotating stars surrounded by a largely rotationally supported (e.g., Hanuschik, these proc.), flat disk. They possess a fast radiatively driven wind, which is probably more pronounced at higher stellar latitudes. Two or more prograde $g$-type modes of the same low degree, $\ell$, and azimuthal order, $m$, and with co-rotating periods of the order of the rotation period (about one day) are excited. (In addition, there may be other modes, incl. $p$-type modes of shorter period and higher degree.) Perhaps, the peak-to-peak temperature variation of several thousand degrees (Maintz et al., Rivinius, these proc.) periodically modulates the ionisation balance in the inner disk, thereby producing periodic $V / R$ variations. Periodic variations in the strength of the circumstellar Balmer jump and rapidly repeating density enhancements in the wind revealing themselves as DACs in UV resonance lines could also result from the modulation of the stellar surface by NRP.

When the traveling waves belonging to modes with sufficiently large amplitude sum interfere constructively, mass is ejected with super-critical rotational velocity. For such an outburst to unfold as observed, magnetic fields may or may not be essential. Through viscous interaction within the ejecta, some particles transfer their angular momentum to others and, therefore, fall back to the star whereas the gainers can eventually merge with the disk. The ejecta initially orbit the star with a "period" of the order of the rotation period. The line emission arising from the ejecta results in V/R variations with that "period", which decrease in amplitude as the distribution of the ejecta is circularized. DACs in UV wind lines may accompany such events.

The more continuous wind may also contribute to the disk. Probably, ablation and dispersal are the more significant wind effects on the disk. If more than two NRP modes beat as described or the amplitudes of the beating modes undergo slow variations, long-term changes in disk size and emission strength would occur as a result of varying outburst intensities. The interaction between new ejecta and the disk may both trigger and quench global one-armed oscillations of the latter, which are observed as slow $V / R$ variations. 


\section{Comparison with Other Early-type Stars}

The comparison of Be stars and Be-like processes in other types of stars (cf. Kaufer, Zickgraf, these proc.) can be mutually useful for their understanding. However, so far this only appears possible in relatively strongly confined areas: Slowly Pulsating B (SPB) stars are the only early-type stars, which are generally accepted to be low-order $g$-mode pulsators (cf. Aerts, these proc.). For some of them several very closely spaced periods have been reported. However, unlike Be stars, their $v \sin i$ rarely exceeds $200 \mathrm{~km} / \mathrm{s}$.

Luminous Blue Variables (LBV's): Their variations are cyclic on timescales consistent with $g$-modes (Lamers et al. 1998). Since there are no confirmed periods, it can only be speculated, whether the lack of regularity is due to the superposition of several modes. Interestingly, LBV's show outbursts.

Other OBA supergiants: NRP has been detected in a fair number of them. However, most periods are shorter than in Be stars and $|m|$ often exceeds 2 significantly. In various objects, the effects of the photospheric pulsation have been traced into the low-velocity regime of the wind (cf. Prinja 1998). Similar effects have been seen in the B0.7 III star $\epsilon$ Per (Gies et al., preprint) and some Be stars (Peters \& Gies, these proc.). Even if the process(es) causing the Be phenomenon was fully developed in these stars, their incomparably much larger radiation pressure would probably prevent the formation of a circumstellar disk. Helium variable stars: One of the proto-types of these stars, the He-rich star HD 37479 (often, unprecisely, also called $\sigma$ Ori E), is sometimes named as the example of a magnetic Be star. However, the line emission does not come from a circumstellar disk, and the pronounced $l p v$ results from the magnetically induced surface abundance inhomogeneity in this oblique magnetic rotator (Groote \& Hunger 1997). Both the $l p v$ and the emission line profiles have nothing in common with most of the other Be stars discussed at this conference (Bolton 1990, A. Reiners et al. 1999: private communications).

Bn stars are rapidly rotating B stars without a record of observed line emission. The second difference w.r.t. to Be stars seems to be the lack of low-order $l p v$ (Penrod 1986, Baade 1987).

From these rather scattered points, it appears that in a parameter space defined by the equatorial rotational velocity and the period of the main low-order NRP mode, Be stars are largely separated from all other early-type stars, with SPB stars being their nearest neighbours. This would further support the idea that rapid rotation and (multiple) low-order NRP are (among) the necessary properties a B star must have in order to appear as a Be star. However, this does not prove that they are also sufficient to explain the Be phenomenon.

\section{Conclusions}

\subsection{What could be learned so far?}

Next to rapid rotation and a disk, low-degree nonradial pulsation is the most commonly observed property of conventional Be stars. It accounts for most of their short-periodic $l p v$. Perhaps the next most important property are already their outbursts. Recent examples show that they may repeat periodically, thereby enabling for the first time discrete star-to-disk mass transfer events to 
be observed in great detail and at all wavelengths. Such periodically repeating outbursts are triggered by the beating of NRP modes with low, identical degree, $\ell$, and identical azimuthal order, $m$. During and following outbursts, additional, transient periodicities may develop in lines formed in the outer photosphere or inner disk. The photospheric temperature variation associated with the NRP may periodically modulate the ionisation balance of the inner disk.

Although squeezed into compact form, only little of the above could have been said just a few years ago. This most visibly underlines what change in substance the term "Be phenomenon" has undergone in a relatively short while. Although this paper is restricted to observed periodic phenomena, even an allencompassing review of the Be phenomenon could hardly deny the growing importance of periodic variabilities on the way towards its understanding.

\subsection{What is not (yet) known?}

This way is still a long one, and whether the path via periodic variabilities is the shortest, or even just a correct one, will only be known at the still unscheduled time of arrival. However, observational results obtained during the past couple of years permit the formulation of a number of useful, concrete questions. They may help shorten the travel (maybe, leading towards unexpected directions!) but are in any event worthwhile answering in their own right:

- Can NRP explain the short-periodic $l p v$ completely, or are there also detectable signatures of co-rotating structures?

- The short-term variability, which seems so important in the earlier spectral sub-types, is largely lacking in the later ones. Is the role of the variability overestimated, or are there different ways to let a B star look like a Be star?

- If all B-type stars within a certain range in mass on the main sequence go through a phase of $g$-mode pulsation, do slow rotators become SPB stars and rapid ones Be stars? In this case, the Be phenomenon would be a common evolutionary phase of all rapidly rotating B stars, not an evolutionary branch depending on additional parameters.

- Is it possible to define the Be phenomenon without a disk, e.g., just by the combination of rapid rotation and multiple low-degree $g$-mode pulsation?

- Are Be stars magnetically distinguished from other early-type stars, especially from the Bn stars?

- What physical process is triggered by the beating of NRP modes so that an outburst results? How is the angular momentum transfer accomplished?

- Are outbursts alone sufficient to build up and maintain a disk?

- To what extent do other stars share the Be phenomenon?

Finally, reliable parallaxes, interferometric measurements of the inclination angle of the disk and, therefore, presumably of the stellar rotation axis, and a further refined limb darkening law are very desirable to further reduce the nonuniqueness of the NRP modelling of the lpv.

Acknowledgments. I thank Thomas Rivinius and Stanislav Štefl for a long, very pleasant collaboration, which led to the presented picture of Be stars. Their and Myron Smith's comments were a great help in finalizing this paper. 


\section{References}

Ando H. 1986, A\&A, 163, 97

Baade D. 1987, in Physics of be Stars, IAU Coll. 92, eds. A. Slettebak \& T.P. Snow, Cambridge Univ. Press, p. 361

Baade D. 1989, A\&A, 222, 200

Baade D. 1992, in Evolutionary Processes in Interacting Binaries, IAU Symp. 151, eds. Y. Kondo, R. Sistero \& R.S. Polidan, Kluwer, Dordrecht, p. 147

Baade D., Balona L.A. 1994, in Pulsation, Rotation and Mass Loss in EarlyType Stars, IAU Symp. No. 162, eds. L.A. Balona, H.F. Henrichs \& J.M.

Le Contel, Kluwer, Dordrecht, p. 311

Balona L.A., Sterken C., Manfroid J. 1991, MNRAS, 252, 93

Balona L.A., Cuypers J., Marang F. 1992, A\&AS, 92, 533

Cook et al. 1995, in Astrophys. Applic. of Stell. Puls., eds. R.S. Stobie \& P.A.

Whitelock, ASP Conf. Ser., No. 83, p. 221

Grady C.A, Bjorkman K.S., Snow T.P. 1987, ApJ, 320, 376

Groote D., Hunger K. 1997, A\&A, 319, 250

Henrichs H.F. 1999, in Variable and Non-spherical Stellar Winds in Luminous Hot Stars, IAU Coll. No. 169, eds. B. Wolf, O. Stahl \& A.W. Fullerton, Springer, Lect. Not. Phys. No. 523, p. 305

Hubert A.M., Floquet M. 1998, A\&A, 335, 565

Kroll P., Hanuschik R.W. 1997, in Accretion Phenomena and Related Outflows, IAU Coll. No. 163, eds. D.T. Wickramasinghe et al., ASP Conf. Ser., No. 121, p. 494

Lamers H.J.G.L.M., Bastiaanse M.V., Aerts C., Spoon H.W.W. 1998, A\&A, 335,605

Osaki Y. 1986, PASP, 98, 30

Osaki Y. 1999, in Variable and Non-spherical Stellar Winds in Luminous Hot Stars, IAU Coll. No. 169, eds. B. Wolf, O. Stahl \& A.W. Fullerton, Springer, Lect. Not. Phys. No. 523, p. 329

Owocki S.P. 1999, in Variable and Non-spherical Stellar Winds in Luminous Hot Stars, IAU Coll. No. 169, eds. B. Wolf, O. Stahl \& A.W. Fullerton, Springer, Lect. Not. Phys. No. 523, p. 294

Penrod, D. 1986, PASP, 98, 35

Peters G.J. 1998, in Cyclical Variability in Stellar Winds, eds. L. Kaper \& A.W. Fullerton, ESO Astrophys. Symp., Springer, p. 127

Prinja R.K. 1998, in Cyclical Variability in Stellar Winds, eds. L. Kaper \& A.W. Fullerton, ESO Astrophys. Symp., Springer, p. 92

Rivinius Th., Baade D., Stefl S., Stahl O., Wolf B., Kaufer A. 1998a, A\&A, 333, 125

Rivinius Th., Baade D., Štefl S., Stahl O., Wolf B., Kaufer A. 1998b, A\&A, 336, 177

Rivinius Th., Štefl S., Baade D., Stahl O., Wolf B., Kaufer A. 1998c, Be Star Newsl., No. 33, p. 15 
Sareyan J.P., Gonzalez-Bedolla S., Guerrero G., Chauville J., Huang L., Hao J.X., Guo Z.H., Adelman S.J., Briot D., Alvarez M. 1998, A\&A, 332, 155

Štefl S., Baade D., Cuypers J. 1995, in Astrophysical Applications of Stellar Pulsation, IAU Coll. No. 155, eds. R.S. Stobie and P.A. Whitelock, ASP Conf. Ser., No. 83, p. 303

Townsend, R.H.D. 1997, PhD thesis, University College, London

Underhill A., Doazan V. 1982, B Stars with and Without Emission Lines, CNRS/

NASA Monogr. Ser. Nontherm. Phen. Stell. Atmosph., NASA SP-456

\section{Discussion}

M. Smith: (i) I've recently become skeptical about the "lighthouse mechanism" for explaining the V/R variations of the HeI lines in Be stars. This attitude comes from simulations of "clouds" with the CIRCUS software. CIRCUS suggests that it's hard to see a "reflection" in a hot line (HeI) from surface temperature modulations unless the cloud is within a few tenths of a $R_{\star}$.

(ii) I'am also skeptical of the characterization of the long-period NRP modes as classical $g$-modes. These modes are unlike those in other stars (e.g., DA white dwarfs) showing $g$-modes because many of them appear over a range of frequencies. Therefore, I find the Lee \& Saio result (1986, MNRAS, 221, 365) appealing that a global $g^{+}$-mode is selected by a $g^{-}$-mode in a rapidly rotating core.

(iii) I believe the "Osaki mechanism" suffers some problems in trying to explain mass ejection purely by NRP. These include: (a) the likely inclusion of retrograde modes observed in at least some Be stars (the mechanism then works to decrease angular momentum), (b) the shocking of waves primarily works simply to dissipate them by heat generation, and (c) Osaki gives no numbers to demonstrate the efficiency of angular momentum or energy transfer through wave shocking. J. Telting: Balona has shown (yesterday) that the occurrence of Be stars may be related to metallicity: the lower the metallicity, the higher the proportion of Be stars. Given that the $\kappa$-mechanism is related to the metallicity, is the above consistent with your suggestion that the Be phenomenon is related to pulsations?

D. Baade: Balona's statement was essentially based on a study by Maeder et al. (1999, A\&A, 346, 459), which compares about 20 clusters of different metallicity and age. However, so long as it is not known whether the Be phenomenon is an evolutionary stage of all rapidly rotating B stars or a separate evolutionary branch, that depends on additional stellar parameters, this is a difficult matter. In any event, many Be stars are nonradial pulsators, and there are strong hints that in at least some of them outbursts are triggered by NRP. Whether outbursts require, e.g., magnetic fields for their full development and outbursts alone are sufficient to make Be stars is yet to be investigated.

Th. Rivinius: I would like to add that, other than for $p$-modes, the $g$-mode instability strip is rather robust against some reduction in metallicity as, e.g., in the Magellanic Clouds.

L. Balona: In our recent series of echelle spectra of $\mu$ Cen we find that greyscale difference spectra are quite different from night to night. If the main periods are around $0.5 \mathrm{~d}$, then the spectra should more or less repeat at the same hour every 
night. We find that the greyscale plots are well represented by a single period of $0.64 \mathrm{~d}$. There appear to be two features on the star which are separated by $0.5 \mathrm{~d}$ which gives rise to the 0.5-d periodicity in the RV curve. One of the features changes intensity over a long timescale giving rise to the amplitude modulations and apparent multiperiodicity when measuring the RV's.

D. Baade: Your data confirm the low summed amplitude of the four 0.5-d variations predicted by our ephemeris (Rivinius et al. 1998c) for your observing run (cf. Baade et al., these proc.). So, your spectra should show a relatively much larger effect of the 0.28 -d variability, and one would not expect the $l p v$ of consecutive nights to look alike. Our spectra, too, show a similar $l p v$ every two days. However, this is just due to the beating of the $0.5-\mathrm{d}$ and $0.28-\mathrm{d}$ periods.

L. Balona: W. Dziembowski and I (1999, MNRAS) have recently investigated the stability of long period modes in models of B0-B3 stars. We find that none of these models show instability with periods typically seen in Be stars.

D. Baade: It is very important indeed to reconcile theory and the interpretation of observations. However, since for many years not even the $\beta$ Cephei stars would pulsate in theoretical models, both theoretical and observational reports need to be critically questioned in the light of the respective other results.

P. Harmanec: You pointed out the basic problems of the Be-star research very well indeed. I confess, however, that I do not share your optimism that most of the phenomena can be described as interference of several periodic phenomena. In your own words: The open question is whether, say, your four closely spaced 0.5 -d periods of $\mu$ Cen represent a "new Kepler law" or just a new application of 'epicycles'. I'm not surprised by numerous reports of multiperiodicity, since every limited time series can be de-composed into a Fourier series of sinusoidal terms. When I tried to analyze either photometric or spectroscopic observations for multiperiodicity, I was indeed finding a number of "periods." But the disturbing thing was that the rms error of the fit was decreasing very slowly with gradually added 'periods'. I noted that also your phase diagrams for $\mu$ Cen show a great deal of scatter. So, the question is: Are we not trying to describe cyclic or even partly stochastic variations by multiple periods?

D. Baade: Our period analysis of $\mu$ Cen (for details see again Baade et al., these proc.) is probably as imperfect as any other one. However, there can be no doubt that it is basically correct and complete. The most important argument is that the four 0.5-d periods were derived from stellar lines, whereas the beat periods, which do not appear in the window function, show up in circumstellar lines (outbursts). I think, one can hardly wish to have a more independent simultaneous confirmation of multiperiodic variations.

R. Townsend: The existence of 'missing' outbursts (i.e. outburst episodes expected but not found) remains compatible with a NRP mode mode beating phenomenon, since significant vibration energy is lost from a star during an outburst, and it may take more than one subsequent beat period before beat amplitudes become large enough for another outburst.

D. Baade: This would be especially true, if there are several beat periods leading to outbursts. All the more remarkable is it that in $\mu$ Cen we have not so far observed an outburst, that failed completely. By contrast, 28 Cyg has so far evidently been much less dependable. 